\section{Prevalência de nascimentos gemelares em Pelotas, Rio Grande do Sul, Brasil}

\section{Prevalence of twin births in Pelotas, in the State of Rio Grande do Sul, Brazil}

\section{Abstract}

Objectives: to evaluate the prevalence of twin births in Pelotas, Rio Grande do Sul, Brazil, during three periods, and the influence of a number of factors on this occurrence.

Methods: a cross-cutting (multiple time series) study was carried out, using the database of the Congenital Defect Monitoring Program, which registers all of the births occurring in the five hospitals of the city of Pelotas, for the years 1993, 1997 and 2003.

Results: the mean prevalence of twin births per thousand births (\%o)t was 8.95\%o (monozygotic $=2.20 \%$ and dizygotic $=6.76 \%$ ). The prevalence of triplets was $0.07 \%$. The total prevalence for twin births, in the case of both monozygotic and dizygotic twins rose over the period studied. The mean age of the mother (27.53 years) and the order in the series of gestations (2.35) were significantly higher in cases of twin births than in cases of the birth of a single infant (26.03 years and 2.14 respectively).

Conclusions: mothers aged $\geq 30$ years were responsible for the increase in the prevalence of twin births in Pelotas. The higher mean position in a sequence of gestations among mothers of twins ruled out the significance of the use of fertility treatment techniques.

Key words Twins, monozygotic, Twins, dizygotic, Maternal age, Birth order, Income
Cesar Fernando Geraldo 1

Gilberto de Lima Garcias 2

Maria da Graça Martino Roth 3

1-3 Laboratório de Genética. Escola de Medicina. Universidade Católica de Pelotas. Rua Félix da Cunha, 412.Pelotas, RS, Brasil. CEP: 96.010-000,E-mail: cezargeraldo@gmail.com

\section{Resumo}

Objetivos: avaliar a prevalência de nascimentos gemelares em Pelotas, $R S$, em três períodos da última década e a influência de alguns fatores sobre este processo.

Métodos: estudo de corte transversal (série temporal), baseado no banco de dados do Programa de Monitorização de Defeitos Congênitos, onde se encontram registrados todos os nascimentos ocorridos nos cinco hospitais da cidade de Pelotas, nos anos de 1993, 1997 e 2003.

Resultados: a taxa média de gêmeos por mil nascimentos foi de 8,95\%o (monozigóticos $=2,20 \%$ e dizigóticos=6,76\%o). A taxa de triplos ficou em 0,07\%. A taxa total de nascimentos gemelares, assim como de gêmeos monozigóticos e dizigóticos sofreu elevação no período. A média de idade (27,53 anos) e da ordem gestacional $(2,35)$ das mães de gêmeos foi significativamente mais elevada do que das mães de únicos $(26,03$ anos e 2,14) respectivamente. Verificaram-se números aproximados de partos gemelares e únicos nos diferentes grupos de renda materna analisados.

Conclusões: as mães com idade maior ou igual a 30 anos foram responsáveis pelo aumento da taxa de gêmeos em Pelotas. A média mais elevada de ordem gestacional em mães de gemelares descartou o uso significativo de técnicas de reprodução assistida.

Palavras-chave, Gêmeos monozigóticos, Gêmeos dizigóticos, Idade materna, Ordem de nascimento, Renda familiar 


\section{Introdução}

Os estudos com gêmeos têm recebido maior ênfase à medida que os avanços tecnológicos fornecem novos conhecimentos sobre o tema. ${ }^{1} \mathrm{O}$ nascimento de gêmeos não é um evento raro; em média, uma em cada 40 crianças que nascem é gêmeo, ${ }^{2}$ porém a taxa de nascimentos gemelares apresenta valores diferentes de acordo com a zigozidade dos pares (monozigóticos ou dizigóticos). Os gêmeos monozigóticos ocorrem como um evento embriológico ao acaso, sem influência ambiental ou determinação genética, não estando o acontecimento desses gêmeos na dependência da raça, estado nutricional, número de partos ou da idade materna. 3,4 Assim, esta taxa tem permanecido praticamente constante na maioria dos países, em torno de 3,5-4,0/1000.5,6

Alguns autores acreditam que o nascimento de gêmeos monozigóticos pode estar relacionado a alguns fatores. O uso da fertilização in vitro e da indução da ovulação elevam o número de gêmeos monozigóticos, por razões ainda desconhecidas. ${ }^{7}$ Os efeitos residuais dos anticoncepcionais orais, levando à diminuição da motilidade tubária e (ou) alterações da mucosa endometrial, e (ou) do epitélio tubário, podem ser os responsáveis pelo aumento da taxa desse tipo de gêmeo, 8 como por exemplo, o observado em mães multigestas acima de 30 anos em estudo realizado na cidade de São Paulo, no Hospital Israelita Albert Einsten. 9

Com relação a gêmeos dizigóticos, seu acontecimento está na dependência de diversos fatores, como: nível socioeconômico, idade materna, paridade, raça, nutrição, hereditariedade, entre outros. 10 A principal causa do aumento verificado na taxa de dizigóticos tem sido o uso da reprodução assistida, 4,11 sendo essa, na verdade, uma taxa artificial. Na Austrália a taxa subiu de 12 para 17/1000 entre 1986 e 1997 e, em relação a trigêmeos, de 0,19 para 0,61/1000, enquanto nos Estados Unidos a taxa de trigêmeos aumentou em seis vezes seu valor. ${ }^{4}$

O acompanhamento da taxa de gêmeos é extremamente importante, principalmente por dois aspectos: o primeiro refere-se ao fato de que a taxa natural de gêmeos dizigóticos pode ser utilizada no estudo da fertilidade humana,6,12-14 sendo considerada uma ferramenta barata, simples e efetiva de monitorar a fertilidade tanto masculina quanto feminina, ${ }^{3}$ uma vez que o acontecimento de gêmeos dizigóticos está na dependência de dupla ovulação, qualidade e quantidade do esperma e no acontecimento do coito no período fértil, entre outros fatores. 6

Levando-se em conta os países onde a repro- dução assistida não é largamente utilizada, observase um declínio na taxa natural de gêmeos dizigóticos 12 e um consenso de que possivelmente a fertilidade humana esteja diminuindo nas últimas décadas. ${ }^{3} \mathrm{O}$ aumento observado na taxa desse tipo de gêmeo, provocado pelo uso das técnicas de reprodução assistida, ${ }^{4}$ pode estar encobrindo o declínio da taxa natural de gêmeos dizigóticos e, conseqüentemente, a queda da fertilidade humana também. ${ }^{3}$

$\mathrm{O}$ segundo aspecto refere-se ao fato de que as gestações múltiplas estão relacionadas com diversas complicações perinatais tanto para as mães como para seus filhos. ${ }^{10} \mathrm{Em}$ todas as populações estudadas, a taxa de mortalidade perinatal, nesse tipo de gestação, é maior do que em gestações únicas 15 e aumenta com o número de fetos. ${ }^{4} \mathrm{~A}$ taxa de mortalidade perinatal é quatro vezes mais alta para gêmeos e seis vezes mais elevada para triplos do que para gestações únicas. $16 \mathrm{Em}$ média, gêmeos pesam 1/3 menos e triplos a metade do peso de únicos. 10

Gêmeos nascem, em média, três semanas antes de únicos 17 e $41,2 \%$ dos triplos nascem antes de completarem 33 semanas. 18 Os gemelares têm uma chance seis vezes maior do que os únicos de apresentarem restrição no crescimento fetal e apresentam um risco duas vezes maior de anemia em relação aos únicos. 4

Os problemas associados a gestações múltiplas não acabam com o parto. Dificuldades da criança relativas à leitura e fala, o déficit de atenção e hiperatividade podem aparecer mais tarde, 4 além do aumento no risco de morbidade até os cinco anos de idade. 19 Podem ainda ocorrer diversas formas de incapacidade, principalmente paralisia cerebral, que tem o risco aumentado de três a sete vezes em gêmeos e dez vezes em triplos. 20

Com relação às mães, a depressão aparece mais comumente em mulheres que tiveram gestações múltiplas. ${ }^{21} \mathrm{O}$ risco de parto por cesária é duas vezes maior para mães de gemelares, assim como o risco de hemorragia antes do parto e pós-parto também é duas vezes mais elevado para essas mães. ${ }^{22} \mathrm{O}$ risco de eclâmpsia e pré-eclâmpsia são quatro e três vezes, respectivamente, mais elevados em gestantes gemelares do que em únicas. 4

Diante das inúmeras complicações advindas da gravidez múltipla e de sua relação com a fertilidade humana, o presente trabalho visa avaliar o comportamento da taxa de nascimentos gemelares na cidade de Pelotas e verificar a influência da idade materna, ordem gestacional e renda familiar sobre este acontecimento, colaborando para o monitoramento da taxa de nascimentos gemelares nessa cidade e despertando a atenção para esse tipo de gestação. 


\section{Métodos}

Foi realizado um estudo de corte transversal (série temporal) com base nos dados registrados no banco de dados do Programa de Monitorização de Defeitos Congênitos do Laboratório de Genética da Escola de Medicina da Universidade Católica de Pelotas, onde se encontram registrados os 16.924 partos ocorridos nos cinco hospitais da cidade de Pelotas: Beneficência Portuguesa, Hospital São Francisco de Paula, Hospital Escola da Fundação de Apoio Universitário, Santa Casa de Misericórdia e Hospital Miguel Piltcher, nos anos de 1993, 1997 e 2003. Foram considerados os dados provenientes de nascidos vivos ou mortos, com peso superior a 500 g. Os fetos com peso igual ou inferior a $500 \mathrm{~g}$, correspondem a idade gestacional de até 22 semanas, foram considerados como abortos. 23

As análises estatísticas dos dados foram realizadas através do programa SPSS 8.0, sendo determinadas as freqüências de nascimentos gemelares e únicos e a associação de fatores maternos (idade da mãe e ordem gestacional) e sócio-demográficos (renda da mãe) com os nascimentos gemelares. Para calcular as razões de prevalência de nascimentos gemelares (com seus intervalos de confiança de $95 \%$ ) em relação à idade materna, ordem gestacional e renda familiar, foi utilizado o programa Epi-Info versão 3.3.2, o valor de " $p$ " considerado como significante foi $<0,05$ e o intervalo de confiança adotado foi $95 \%$. O teste qui-quadrado foi usado para comparação de freqüências de idades e ordens gestacionais entre os grupos de mães de únicos e gemelares.

A freqüência de gêmeos dizigóticos e monozigóticos foi estimada pelo clássico método diferencial de Weinberg, 24 uma vez que nenhum teste de zigozidade foi realizado. Por esse método estima-se a taxa de gêmeos dizigóticos, duplicando o número de pares de gêmeos discordantes quanto ao sexo. A taxa de monozigóticos é obtida pela diferença entre o número total de gêmeos e o número de pares dizigóticos estimados.

As taxas de nascimentos gemelares foram expressas referindo-se ao número de nascimentos gemelares 'a cada' 1000 nascimentos totais ocorridos.

\section{Resultados}

Dos 16.924 partos ocorridos nos três anos em estudo (1993, 1997 e 2003), 16.774 foram de únicos, 149 de gêmeos e um de trigêmeos (Tabela 1), sendo que, nesse período, houve uma redução progressiva no número de partos na cidade de Pelotas. Foram registrados 6281 partos no ano de 1993, $5691 \mathrm{em}$ 1997 e 4952 em 2003. Por outro lado, o número de partos gemelares aumentou, a cada ano: 46 em 1993, 48 em 1997 e 56 em 2003 (Figura 1).

A taxa média de nascimentos gemelares 'por cada' mil nascimentos (\%) foi de 8,95 no período estudado. A variação no período foi de 51,77\%, aumentando de 7,32\%o em 1993 para $11,11 \%$ em 2003, mostrando ter influenciado positivamente a variação temporal no período. As taxas médias de gêmeos dizigóticos e monozigóticos foram de $6,76 \%$ e $2,20 \%$, respectivamente, sendo que ambas sofreram aumento no período. Apenas uma gestação tripla foi registrada, ficando sua taxa média em 0,07\% (Tabela 1).

Com relação à renda familiar, ambos os grupos de mães, de únicos e de gemelares, apresentaram percentuais de partos aproximados nos grupos de renda materna avaliados. A grande maioria das mães, mais de $90 \%$, se encontravam nas faixas de renda de até cinco salários mínimos. Os resultados do cálculo da razão de prevalência, com relação à gemelaridade e a renda familiar, não apresentaram resultados significativos (Tabela 2).

A média da idade materna para mães de gêmeos [27,53 anos (DP 6,31)] foi significativamente mais elevada do que para mães de únicos [26,03 anos (DP $6,65)]$. Durante o decorrer da década em estudo, as médias de idade materna, tanto para mães de únicos como de gemelares sofreram aumento. A média de idade para mães de únicos foi de 26,01 anos em 1993 e 26,11 em 2003, enquanto nas mães de gêmeos o aumento foi maior: 27,48 anos em 1993 e 28,26 em 2003. A análise da razão de prevalência mostrou que o risco de gravidez gemelar foi diretamente proporcional à idade materna, excluindo-se o último grupo de idade ( $\geq 42)$, no qual não foi registrado nenhum caso desse tipo de gestação (Tabela 3 ).

No grupo de mães de $<30$ anos, o número de partos gemelares, por ano, se comportou de maneira praticamente estável, enquanto no grupo de mães de $\geq 30$ anos o número de partos gemelares aumentou a cada ano. As mães com idades $\geq 30$ anos obtiveram um percentual bem maior de partos gemelares $(1,2 \%)$ em relação às mães de $<30$ anos $(0,7 \%)$ (Tabela 4).

Com relação à zigozidade dos gemelares, as mulheres na faixa de 30 a 35 anos apresentaram um percentual maior de gêmeos monozigóticos $(62,5 \%)$ do que de dizigóticos $(37,5 \%)$ nos dois últimos anos avaliados, enquanto as mulheres de 36 a 41 anos tiveram somente dizigóticos em todo o período.

Obteve-se uma média de ordem gestacional 
Tabela 1

Número de partos ocorridos nos três anos de estudo (1993, 1997 e 2003) na cidade de Pelotas, Rio Grande do Sul.

\begin{tabular}{|c|c|c|c|c|c|c|c|c|c|}
\hline \multirow[t]{2}{*}{ Ano } & \multirow[t]{2}{*}{ Partos Únicos } & \multicolumn{4}{|c|}{ Gemelares } & \multicolumn{4}{|c|}{ Taxa por 1.000 nascimentos } \\
\hline & & DZ & $\mathrm{MZ}$ & Triplos & Total & DZ & MZ & Total & Triplos \\
\hline 1993 & 6.235 & 34 & 12 & 0 & 46 & 5,41 & 1,91 & 7,32 & 0 \\
\hline 1997 & 5.643 & 34 & 14 & 0 & 48 & 5,97 & 2,46 & 8,43 & 0 \\
\hline 2003 & 4.896 & 44 & 11 & 1 & 56 & 8,89 & 2,22 & 11,11 * & 0,20 \\
\hline Total & 16.774 & 112 & 37 & 1 & 150 & 6,76 & 2,20 & 8,95 & 0,07 \\
\hline
\end{tabular}

DZ=dizigóticos; MZ=monozogóticos; Teste $\chi^{2}$ : 1993 × 1997 (NS); 1997 × 2003 (NS); 1993 x 2003; p=0,027.

significativamente mais elevada para gemelares

Figura 1

Número total de partos e de partos gemelares ocorridos nos três momentos do estudo.

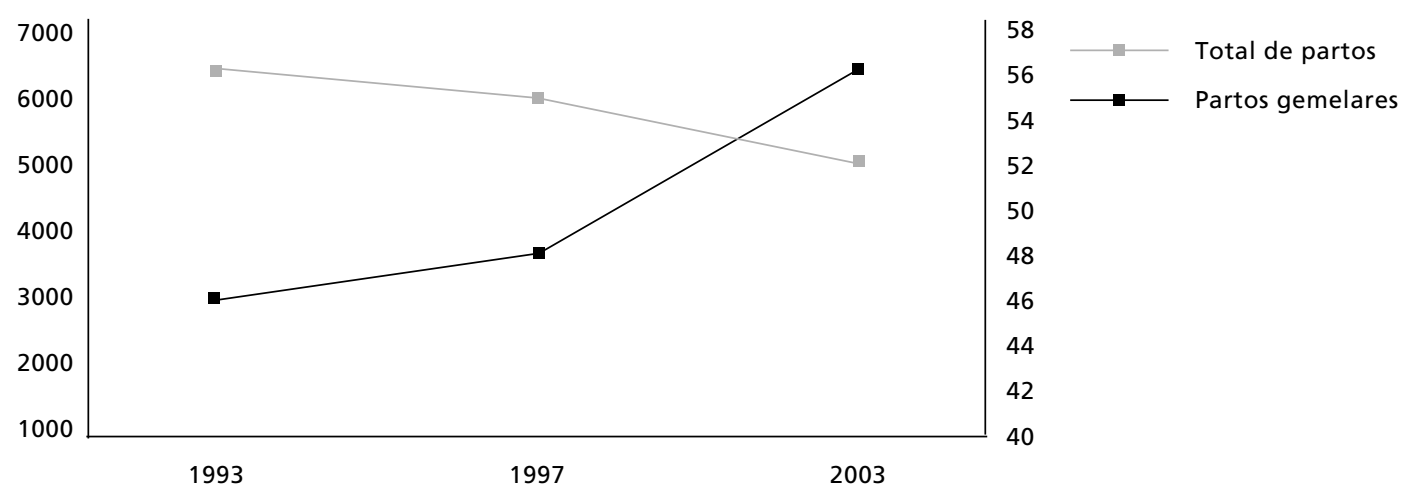

Tabela 2

\begin{tabular}{lccccccc}
\hline \multicolumn{7}{l}{ Razão de prevalência da freqüência de gemelaridade em relação à renda familiar. } \\
\hline Renda familiar (SM) & Partos únicos & $\%$ & Partos gemelares & $\%$ & RP & IC95\% & $\boldsymbol{p}$ \\
\hline 1 ou $<$ & 4.817 & 31.6 & 40 & 30,5 & 0,90 & $0,51-1,58$ & $\mathrm{~ns}$ \\
$1,1-3$ & 6.240 & 41,0 & 59 & 45,0 & 1,02 & $0,61-1,74$ & $\mathrm{~ns}$ \\
$3,1-5$ & 2.275 & 15,0 & 21 & 16,0 & 1,0 & & \\
$5,1-10$ & 1.397 & 9,0 & 6 & 4,6 & 0,47 & $0,17-1,22$ & $\mathrm{~ns}$ \\
$>10$ & 519 & 3,4 & 5 & 3,9 & 1,04 & $0,34-2,94$ & $\mathrm{~ns}$ \\
Total & 15.248 & 100,0 & 131 & 100,0 & & & \\
\hline
\end{tabular}

$\mathrm{RP}=$ razão de prevalência; ns=não significante; $\mathrm{IC}=$ intervalo de confiança; $S M=$ salário mínimo.

[2,62 (SD 1,72)] do que para únicos [2,38 (SD $1,68)]$. A média de ordem gestacional para mães de gemelares aumentou em $6,9 \%$, enquanto para mães de únicos houve uma queda de $2,3 \%$ no período. $\mathrm{O}$ cálculo da razão de prevalência mostrou elevação do risco de parto gemelar de acordo com a elevação da ordem gestacional até a $3^{\mathrm{a}}$ ordem (Tabela 5). 
Tabela 3

\begin{tabular}{|c|c|c|c|c|c|c|c|}
\hline Idade materna (anos) & Partos únicos & $\%$ & Partos gemelares & $\%$ & $\mathbf{R P}$ & IC95\% & $p$ \\
\hline$\leq 19$ & 3.078 & 18,5 & 16 & 10,7 & 1,0 & & \\
\hline $20-24$ & 4.502 & 27,1 & 37 & 24,8 & 1,58 & $0,85-2,97$ & ns \\
\hline $25-29$ & 3.990 & 24,0 & 34 & 22,7 & 1,64 & $0,87-3,11$ & ns \\
\hline $30-35$ & 3.383 & 20,4 & 43 & 29,0 & 2,45 & $1,33-4,53$ & 0,001 \\
\hline $36-41$ & 1.457 & 8,8 & 19 & 12,8 & 2,51 & $1,23-5,13$ & 0,005 \\
\hline$\geq 42$ & 193 & 1,2 & - & - & 0,00 & $0,00-5,10$ & ns \\
\hline Total & 16.603 & 100,00 & 149 & 100,0 & & & \\
\hline
\end{tabular}

$\mathrm{RP}=$ razão de prevalência; ns=não significante; IC=intervalo de confiança.

Tabela 4

Razão de prevalência da freqüência de gemelaridade em relação a grupos de idade materna.

\begin{tabular}{lccccccc}
\hline Idade materna & Partos únicos & $\%$ & Partos gemelares & $\%$ & RP & IC95\% & $\boldsymbol{p}$ \\
\hline$<30$ & 11.570 & 69,5 & 87 & 58,3 & 1.0 & & \\
$\geq 30$ & 5.033 & 30,5 & 62 & 41,7 & 1,64 & $1,17-2,30$ & 0,003 \\
Total & 16.603 & 100,0 & 149 & 100,0 & & & \\
\hline
\end{tabular}

$R P=$ razão de prevalência; ns=não significante; $I C=$ intervalo de confiança; $X^{2}=8,90$.

Tabela 5

Razão de prevalência da freqüência de gemelaridade em relação à ordem gestacional.

\begin{tabular}{lccccccc}
\hline Ordem gestacional & Partos únicos & $\%$ & Partos gemelares & $\%$ & RP & IC95\% & $\boldsymbol{p}$ \\
\hline Primeira & 6.271 & 38,1 & 44 & 29,9 & 1,0 & \\
Segunda & 4.494 & 27,3 & 39 & 26,5 & 1,24 & $0,79-1,95$ & $\mathrm{~ns}$ \\
Terceira & 2.721 & 16,5 & 32 & 21,8 & 1,68 & $1,04-2,71$ & $\mathrm{~ns}$ \\
$\geq$ quarta & 2.980 & 18,1 & 32 & 21,8 & 1,53 & $0,95-2,47$ & $\mathrm{~ns}$ \\
Total & 16.466 & 100,0 & 147 & 100,0 & & & \\
\hline
\end{tabular}

$\mathrm{RP}=$ razão de prevalência; ns=não significante; IC=intervalo de confiança..

\section{Discussão}

A taxa média de nascimentos gemelares encontrada $(8,95 \%)$ pode ser considerada como a taxa natural de nascimentos gemelares para a cidade de Pelotas, na última década, uma vez que os registros de nascimentos abrangeram a população em geral. Em nível de Brasil, esta taxa foi semelhante às encontradas na região sudeste, $8,8 \%{ }_{0} 12$ e 7,99\%, 25 consideradas como taxas naturais, porém ficou bem abaixo da registrada em trabalho envolvendo mulheres de classe alta $(24,02 \%),{ }^{9}$ possivelmente pelo uso de técnicas de reprodução assistida.
A taxa de dizigóticos $(6,76 \%$ ) ficou acima da média da população, no geral, do sudeste brasileiro, que é de 4,72\%. ${ }^{12}$ Esse valor pode ser considerado como a taxa média natural de gêmeos dizigóticos mais elevada, nas últimas décadas, no Brasil. Entretanto, fica abaixo da taxa natural de $10 \%$ citada para a Grã-Bretanha e América do Norte 26 e bem abaixo da taxa verificada em um estudo feito em um hospital com alto padrão de cuidados reprodutivos $(19,51 \%)$, na cidade de São Paulo, ${ }^{9}$ onde mais comumente são utilizadas técnicas de reprodução assistida.

Em Pelotas, a taxa de média de monozogóticos 
em Pelotas (2,20\%o) ficou abaixo da taxa média mundial $(3,5$ a 4,0\%o).2,6 Taxas maiores também foram encontradas no Brasil $(4,1 \%)$ em amostra geral da população12 e em amostra de classe socioeconômica alta (4,5\%).9 O aumento de gêmeos de sexos distintos (30\%) em 2003 colaborou para a baixa média de monozigóticos, visto que os monozigóticos são calculados pela diferença entre o número total de gemelares e o dobro do número de gêmeos de sexos distintos. Apenas uma gestação tripla foi registrada, a qual ocorreu no ano de 2003, ficando sua taxa média em $0,07 \%$. No Brasil, a taxa média é de 0,15 para a população em geral da região sudeste. ${ }^{12}$

Desde que, conforme a renda, tanto as mães de gêmeos como de únicos mostraram percentuais bastante aproximados de partos, pode-se concluir que a renda familiar não influenciou as taxas de nascimentos gemelares. Como mais de $90 \%$ das mães possuíam renda de até cinco salários mínimos, não se observou o efeito da reprodução assistida sobre a taxa de gemelaridade, uma vez que devido à situação econômica dessa população, essa é uma prática ainda muito pouco utilizada, pois trata-se de técnica de custo elevado.

A média de idade materna mais elevada para mães de gêmeos do que para mães de únicos, encontrada neste trabalho, é apoiada pela literatura, já que a idade materna é positivamente correlacionada com a taxa gemelar.12,27 Esse fato é reforçado pela obser-

\section{Referências}

1. Hall JG. Twinning. Lancet. 2003; 362: 735-43.

2. Nylander PPS. Frequency of multiple births. In: MacGillivray I, Nylander PPS, Corney G. editors. Human multiple reproduction. London: W. B. Saunders; 1975. p. 87-98.

3. Tong S, Short RV. Dizygotic twinning as a measure of human fertility. Hum Reprod. 1998; 1: 95-8.

4. Umstad MP, Gronow MJ. Multiple pregnancy: a modern epidemic? Med J Aust. 2003; 178: 613-5.

5. Bulmer MG. The Biology of Twinning in Man. Oxford: Clarendon; 1970.

6. James WH. Second survey of secular trends in twinning rates. J Biosoc Sci. 1982; 14: 481-97.

7. Derom C, Vlietinck R, Derom R, Van den Berghe H, Thiery $\mathrm{M}$. Increased monozigotic twinning rate after ovulation induction. Lancet.1987; 1: 1236-8.

8. Beiguelman B. O estudo de gêmeos. São Paulo: E-book; 2003. [Publicação eletrônica da Sociedade Brasileira de Genética] Acesso em: 20 fev. 2008. Disponível em: http://www.sbg.org. br/ebooks.html.

9. Colletto GMDD, Segre CAM, Beiguelman B. Twinning rate in a Southeastern Brazilian population with a high standard of reproductive care. São Paulo Med J. 2001; 119: 216-9. vação do cálculo da razão de prevalência, o qual mostra que o risco de gravidez gemelar foi diretamente proporcional à idade materna, excluindo-se o último grupo de idade $(\geq 42)$, no qual não foi registrado nenhum caso desse tipo de gestação.

Embora se tenha observado um aumento da razão de prevalência de partos gemelares, a cada ano do estudo, nas mães com 30 anos ou mais, não se pode descartar a influência das mães com idade abaixo de 30 anos na elevação da taxa de nascimentos gemelares, pois elas mantiveram um número praticamente estável de partos gemelares na década, apesar da diminuição do número de partos únicos.

Os resultados aqui apresentados demonstram claramente o aumento de nascimentos de gêmeos na população de Pelotas e sugerem que se aprofunde a investigação, no sentido de identificar as causas reais desse aumento e, com isso, buscar compreender quais as implicações desse fenômeno no que se refere ao padrão reprodutivo dessa população.

\section{Agradecimentos}

Agradecemos as valiosas colaborações das Professoras Elaine Tomasi (UCPel), por sua contribuição na análise estatística dos dados e Gloria Maria Duccini Dal Colleto (USP) por suas informações a respeito da gemelaridade no Brasil.
10. National Center for Health Statistics. Birth statistics. 1999. [monograph online]. [consulte on 2004 Dez 10]. Available from: http://www.orgsites.com. [2004 Dez 10.

11. Vilska S, Tiitinen A, Hydén-Granskog C, Hovatta O. Elective transfer of one embryo results in an acceptable pregnancy rate and eliminates the risk of multiple birth. Hum Reprod. 1999; 14: 2392-5.

12. Beiguelman B, Franchi-Pinto C, Krieger H, Magna LA. Twinning rate in southeastern Brazilian population. Acta Genet Med Gemelol. (Roma). 1996; 45: 317-24.

13. James WH. Secular trends in monitors of reproductive hazard. Hum Reprod. 1997; 12: 417-21.

14. Lazar P, Hemon D, Berger C. Twinning rate and reproductive faillures. In: Nance WE, editor. Twin research: biology and epidemiology. New York: A.R. Liss; 1977. p. 125-32.

15. Lumme RH, Saarkoski SV. Perinatal deaths in twin pregnancy: a 22-year review. Acta Genet Med Gemelol. (Roma). 1988; 37: 47-54.

16. ESHRE (European Society of Human Reproduction and Embryology) Capri Workshop Group. Multiple gestation pregnancy. Hum Reprod. 2000; 7: 1856-64.

17. Beiguelman B, Franchi-Pinto C, Magna LA. Biological and social traits associated with twinning among Caucasoides and Negroides. Braz J Genet. 1997; 20: 311-8. 
18. Keith LG, Oleszczuk JJ, Keith DM. Multiple gestation: reflections on epidemiology, causes and consequences. Int J Fertil Womens Med. 2000; 45: 206-14.

19. Baird J, Osmond C, Bowes I, Phillips DI. Mortality from birth to adult life: a longitudinal study of twins. Early Hum Dev. 1998; 53: 73-9.

20. Petterson B, Stanley F, Henderson D. Cerebral palsy in multiple births in Western Australia. Am J Med Genet. 1990; 37: 346-51.

21. Thorpe K, Golding J, MacGillivray I, Greenwood R. Comparison of prevalence of depression in mothers of twin and mothers of singletons. BMJ. 1991; 302: 875-8.

22. Riley M, Halliday J. Births in Victoria 1999-2000. Melbourne: Department of Human Services; 2001.

23. Belitzki R, Fescina R, Ucieda F. Definiciones y terminologias aplicables al periodo perinatal: recomendaciones de la Organización Mundial de la Salud y modificaciones de la FIGO. Pub Científica del CLAP. 1978; 757: 136-47.
24. Weinberg W. Beiträge zur hysiologie und pathologie der mehrlingsgeburten beim Menschen. Pflügers Arch. 1901; 88: 346-430.

25.Colletto GMDD, Segre CA, Rielli ST, Rosário H. Multiple birth rates according to different socioeconomic levels: an analysis in four hospitals from the city of São Paulo, Brazil. Twin Res. 2003; 6: 177-82.

26.Nylander PPS. The factors that influence twinning rates. Acta Genet Med Gemelol.(Roma). 1981; 30: 189-202.

27.Allen G, Parisi P. Trends in monozygotic twinning rates by maternal age and parity. Further analysis of Italian data, 1949-1985, and rediscussion of US data, 1964-1985. Acta Genet Med Gemelol. (Roma). 1990; 39: 317-28.

Recebido em 26 de abril de 2006

Versão final apresentada em 23 de julho de 2008

Aprovado em 14 de agosto de 2008 